\title{
The Thirring model as an approximation to the theory of two-photon propagation
}

\author{
A M Kamchatnov†, H Steudel $\ddagger$ and A A Zabolotskii $\S$ \\ $\dagger$ Institute of Spectroscopy, Russian Academy of Sciences, Troitsk, Moscow Region 142092, \\ Russia \\ $\ddagger$ Institut für Physik der Humboldt-Universität, Arbeitsgruppe 'Nichtklassische Strahlung', \\ Rudower Chaussee 5, D-12484 Berlin, Germany \\ $\S$ Institute of Automation and Electrometry, Siberian Branch of Russian Academy of Sciences, \\ 630090 Novosibirsk, Russia
}

Received 30 April 1997, in final form 14 August 1997

\begin{abstract}
We consider the two-photon propagation process under the two-fold approximation that (i) the excitation of the upper level of medium molecules remains small during the process, (ii) the intensity of one of the two participating electromagnetic waves is always small compared to that of the other wave. It is then shown that in the limit of the strong dynamical Stark effect the equations describing this process can be reduced to the classical Thirring model equations. This reduction permits one to develop a much simpler theory of wave interaction than was possible in the case of the original equations. As an example, the periodic solutions of twophoton propagation are considered and the Whitham modulational equations are obtained. The different forms of $U-V$ pairs for the Thirring model are discussed in the appendix. Analogous theory can be developed for the stimulated Raman scattering process.
\end{abstract}

\section{Introduction}

The propagation of light waves in a resonant medium has been widely investigated, both experimentally and theoretically. In particular, two-photon propagation (TPP) and stimulated Raman scattering (SRS), i.e. the coherent propagation of two light beams under the condition of a second-order resonance, have been the focus of considerable theoretical interest (see, e.g. the review article [1]). Currently the progress in optical techniques has advanced to the point where atomic inversion can be achieved by means of coherent TPP [2] or SRS [3] techniques. All this is evidence that further theoretical progress became topical.

An important property of TPP and SRS equations is their integrability in the sense of the inverse scattering transform (IST) method first established in [4,5]. This permits one to obtain exact soliton [5], multisoliton [6], and periodic [7-10] solutions of these equations and investigate some particular cases of evolution of pulses which are short compared with the relaxation and inhomogeneous broadening times. Clearly the two-photon processes of TPP or SRS types might be enhanced by several orders of magnitude when a third level is in a near-to-resonance configuration, cf figure 1. According to numerical estimations given in [5], a few $\mathrm{MW} \mathrm{cm}{ }^{-2}$ are enough to generate soliton-type structures in the subnanosecond region. For such TPP experiments potassium-where non-degenerate two-photon selfinduced transparency was already found in 1972 [11]—would be a possible 


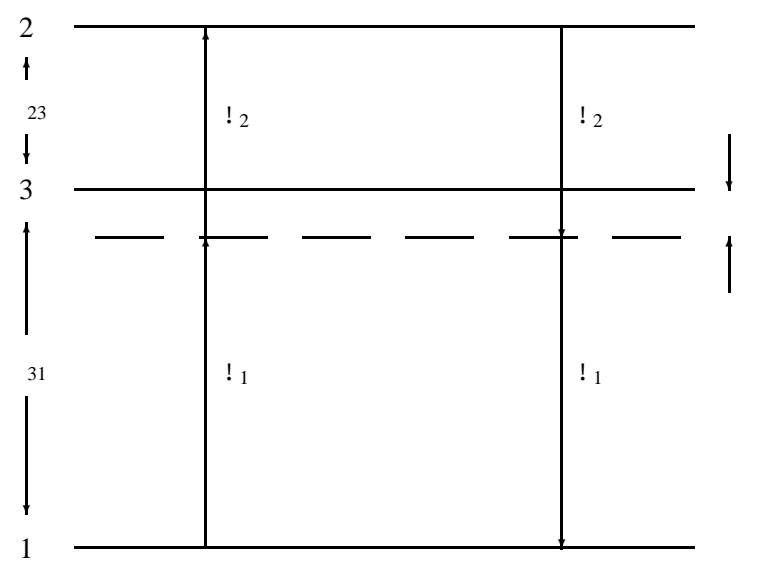

Figure 1. Scheme of transitions in a three-level system in the case of two-photon propagation. Electromagnetic field frequencies $\omega_{1}$ and $\omega_{2}$ resonate approximately with the atomic transitions $3 \rightarrow 1$ and $2 \rightarrow 3$ (frequencies $\Omega_{31}$ and $\Omega_{23}$, respectively), which leads to two-photon emission and absorption processes between levels 1 and 2 .

candidate while, with respect to analoguos SRS experiments, one may have stimulated electronic Raman scattering in indium, in mind [3].

In this paper we wish to note that, in the limit when one electromagnetic wave is much stronger than the other and the inversion of the upper level of the medium is low, the TPP and SRS equations can be reduced to the classical massive Thirring model equations [12], if the coupling constant that describes both the dynamical Stark effect and a populationdependent refraction index is large enough. The Thirring model equations are much simpler than the TPP and SRS equations, so the nonlinear processes can be investigated more easily and in greater detail. These conditions of different magnitudes of wave intensity and low inversion of the upper level may be met in experiment, and this simplification of the theory may become important from a practical point of view. For simplicity, we shall only consider here the TPP case, since the calculations for the SRS case are quite analogous.

Before going on to the formal treatment, let us show that the limit under consideration is quite reasonable from a physical point of view, in particular, for such a three-level system as that mentioned above and shown schematically in figure 1 . We consider the propagation of two electromagnetic waves with electric field envelopes $E_{1}$ and $E_{2}$ and frequencies $\omega_{1}$ and $\omega_{2}$ in a three-level system where electric dipole transitions $2 \rightarrow 3,3 \rightarrow 1$ are allowed and $\Omega_{23}, \Omega_{31}$ denote the exact (one-photon) resonant frequencies. Then, we assume that

$$
\Omega_{31}-\omega_{1}=\omega_{2}-\Omega_{23}=\delta \omega \quad|\delta \omega| \ll \Omega_{31}, \Omega_{23}
$$

such that the two waves are at exact resonance with respect to a two-quantum transition between levels 1 and 2 .

In physical units, the Maxwell equations reduce in a slowly varying envelope approximation to (see, e.g., [5])

$$
\begin{aligned}
& \frac{\partial E_{1}}{\partial x}+\frac{1}{c} \frac{\partial E_{1}}{\partial t}=-\mathrm{i} b_{21}^{1} R_{3} E_{1}+\mathrm{i} k E_{2}^{*} R_{-} \\
& \frac{\partial E_{2}}{\partial x}+\frac{1}{c} \frac{\partial E_{2}}{\partial t}=-\mathrm{i} b_{21}^{2} R_{3} E_{2}+\mathrm{i} k E_{1}^{*} R_{-} .
\end{aligned}
$$

Here $c$ denotes the group velocity which is assumed to be the same at both frequencies. The Bloch vector $R=\left(R_{1}, R_{2}, R_{3}\right), R_{ \pm}=R_{1} \pm \mathrm{i} R_{2}$, describes an effective two-level system 
with levels 1 and 2 and satisfies the following equations of motion

$$
\begin{aligned}
\frac{\partial R_{+}}{\partial t} & =2 \mathrm{i}\left(b_{21}^{1}\left|E_{1}\right|^{2}+b_{21}^{2}\left|E_{2}\right|^{2}\right) R_{+}+2 \mathrm{i} k E_{2}^{*} E_{1}^{*} R_{3} \\
\frac{\partial R_{3}}{\partial t} & =\mathrm{i} k\left(E_{1} E_{2} R_{+}-E_{1}^{*} E_{2}^{*} R_{-}\right) .
\end{aligned}
$$

The terms containing the constants $b_{21}^{F}\left(F=1,2\right.$ is an index of the wave $\left.E_{F}\right)$ describe either an inversion-dependent susceptibility in equation (2) or the dynamical Stark effect in (3), and $k$ is the coupling constant corresponding to two-photon transitions. Quantum mechanical perturbation theory leads to the following expressions for these constants (see $[5,13])$

$$
\begin{aligned}
& b_{21}^{1}=-\frac{\hbar}{\varepsilon_{0}} \frac{\left|k_{13}^{1}\right|^{2}}{\lambda_{1}} \frac{1}{\delta \omega} \\
& b_{21}^{2}=-\frac{\hbar}{\varepsilon_{0}} \frac{\left|k_{23}^{2}\right|^{2}}{\lambda_{2}} \frac{1}{\delta \omega} \\
& k=\frac{2 \hbar}{\varepsilon_{0}} \frac{k_{23}^{2} k_{31}^{1}}{\sqrt{\lambda_{1} \lambda_{2}}} \frac{1}{\delta \omega}
\end{aligned}
$$

where $k_{p q}^{F}$ are the dipole matrix elements of the transitions between levels $p$ and $q$, and $\lambda_{F}=\omega_{F} / c$ is the wavelength of the corresponding electromagnetic wave. As shown in [5], the only essential dimensionless parameter in this problem is the relative dynamical Stark shift coefficient

$$
\Delta=\left(b_{21}^{1}+b_{21}^{2}\right) / k
$$

and estimations (4) lead to the following inequality for its absolute value

$$
|\Delta|=\frac{f_{31} / \lambda_{1}+f_{23} / \lambda_{2}}{2 \sqrt{f_{31} f_{23} / \lambda_{1} \lambda_{2}}} \geqslant 1
$$

where we have introduced more common oscillator strengths $f_{p q} \propto\left|k_{p q}^{F}\right|^{2}$ of the transitions. Actually, the Thirring approximation corresponds to the first essential terms in the series expansion of soliton formulae with respect to powers of small parameter $1 / \Delta^{2}$, which, as we see from inequality (6), can be achieved in the case of large enough asymmetry in the values of $f_{31} / \lambda_{1}, f_{23} / \lambda_{2}$. This justifies an application of the Thirring model approximation to TPP and SRS optical problems.

The Thirring model approximation is also rather interesting from a mathematical point of view. It is well known that integrability is a very subtle property of an equation and can easily be violated by any approximation. For example, an integrable sine-Gordon equation $u_{t t}-u_{x x}+\sin u=0$ loses its integrability under the Klein-Gordon approximation $u_{t t}-u_{x x}+u-u^{3} / 6=0$ correct at $|u| \ll 1$. Naturally, TPP and SRS equations become nonintegrable in analogous approximations but TPP and SRS equations have an additional parameter $\Delta$, and integrability of their approximation is restored in the limit $|\Delta| \gg 1$. This observation reveals new interconnections between different classes of equations and is worthy of thorough investigation.

In the next section we investigate the limit of the TPP equations mentioned above. We find that this limit leads to a new form of the $U-V$ pair for the Thirring model which is different from the standard one $[14,15]$. This ambiguity in the $U-V$ pairs in the Thirring model is considered in the appendix by means of the prolongation method. The last section of the paper is devoted to the derivation of the periodic solutions of the Thirring model equations by means of the finite-gap integration method (see, e.g., [16], 
[17]). We find that the modification of this method suggested in [18] and applied later to a number of integrable equations [19] permits us to obtain periodic solutions in a form suitable for physical applications. In addition, the Whitham equations describing the evolution of modulated periodic solutions are obtained in a convenient form. These results form a basis for applications of the developed theory to concrete physical problems.

\section{The reduction of the TPP equations to the Thirring model equations}

As was mentioned in the introduction, for simplicity we consider only the TPP case. The SRS case can be considered in the same way with minor changes. The TPP equations describe the propagation of two electromagnetic waves with frequencies $\omega_{1}$ and $\omega_{2}$ and electric fields envelopes $E_{1}$ and $E_{2}$ interacting with a two-level system where the energy separation is just $\hbar\left(\omega_{1}+\omega_{2}\right)$ and a two-photon transition is allowed between both these levels. The field amplitudes might be scaled such that $E_{1} E_{1}^{*}$ and $E_{2} E_{2}^{*}$ are the respective photon current densities. The equations acquire a rather symmetric form if we introduce the vector $\boldsymbol{S}$ with components [5]

$S_{1}=\left(E_{1}^{*} E_{2}^{*}+E_{2} E_{1}\right) / I \quad S_{2}=\mathrm{i}\left(E_{1}^{*} E_{2}^{*}-E_{2} E_{1}\right) / I \quad S_{3}=\left(E_{1} E_{1}^{*}+E_{2} E_{2}^{*}\right) / I$

where $I=I\left(t^{\prime}\right)=E_{1} E_{1}^{*}-E_{2} E_{2}^{*}$ is the difference between the two-photon current densities, which is a function of the retarded time $t^{\prime}=t-x / c$ only ( $x$ is a space coordinate along which the waves propagate and $c$ is their group velocity). We assume that $I\left(t^{\prime}\right)$ is always positive. Now we may introduce

$$
\tau=k \int_{t_{0}}^{t} I\left(t^{\prime}\right) \mathrm{d} t^{\prime}
$$

as a new variable instead of $t^{\prime}$, where $k$ is the coupling constant for the two-photon interaction process. If we also introduce the dimensionless space coordinate $\xi$ and the Bloch vector $\boldsymbol{R}$ describing the state of the medium $\left(R_{ \pm}=R_{1} \pm \mathrm{i} R_{2}\right.$ correspond to nondiagonal elements of the density matrix and $R_{3}$ to the difference between populations of the upper and lower levels of the molecules), then the TPP equations take the form $[4,5]$

$$
\begin{aligned}
\frac{\partial R_{+}}{\partial \tau} & =\mathrm{i}\left(\Delta R_{+} S_{3}+R_{3} S_{+}\right) & \frac{\partial R_{3}}{\partial \tau} & =\frac{\mathrm{i}}{2}\left(R_{+} S_{-}-R_{-} S_{+}\right) \\
\frac{\partial S_{+}}{\partial \xi} & =\mathrm{i}\left(\Delta S_{+} R_{3}-S_{3} R_{+}\right) & \frac{\partial S_{3}}{\partial \xi} & =\frac{\mathrm{i}}{2}\left(S_{+} R_{-}-S_{-} R_{+}\right)
\end{aligned}
$$

where $S_{ \pm}=S_{1} \pm \mathrm{i} S_{2}$ and $\Delta$ is the relative dynamic Stark shift coefficient (see (5)). The vectors $\boldsymbol{R}$ and $\boldsymbol{S}$ are normalized according to the conditions

$$
R_{1}^{2}+R_{2}^{2}+R_{3}^{2}=1 \quad-S_{1}^{2}-S_{2}^{2}+S_{3}^{2}=1 .
$$

In the limit of low population of the upper level we have $R_{3} \simeq-1$, that is $\left|R_{ \pm}\right| \ll 1$ and hence

$$
R_{3}=-\sqrt{1-R_{+} R_{-}} \simeq-1+\frac{1}{2} R_{+} R_{-} .
$$

Now let us suppose that the field strength of one field is much less than that of the other,

$$
\left|E_{2}\right| \ll\left|E_{1}\right| \text {. }
$$

Then $S_{+}=2 E_{1} E_{2}$ and $S_{-}=2 E_{1}^{*} E_{2}^{*}$ are small compared to $S_{3}=\left|E_{1}\right|^{2}+\left|E_{2}\right|^{2}$ and consequently

$$
S_{3}=\sqrt{1+S_{+} S_{-}} \simeq 1+\frac{1}{2} S_{+} S_{-} .
$$


On substituting the approximations (10) and (11) into equations (8), we obtain the following system of two equations

$$
\begin{aligned}
& -\mathrm{i} \frac{\partial R_{+}}{\partial \tau}=\Delta R_{+}-S_{+}+\frac{\Delta}{2} S_{+} S_{-} R_{+}+\frac{1}{2} R_{+} R_{-} S_{+} \\
& \mathrm{i} \frac{\partial S_{+}}{\partial \xi}=\Delta S_{+}+R_{+}-\frac{\Delta}{2} R_{+} R_{-} S_{+}+\frac{1}{2} S_{+} S_{-} R_{+} \cdot
\end{aligned}
$$

The second pair of equations (8) is satisfied automatically as a consequence of (12). The first terms on the right-hand sides of equations (12) can be eliminated by means of the substitutions

$$
S_{+} \rightarrow S_{+} \exp [\mathrm{i} \Delta(\xi-\tau)] \quad R_{+} \rightarrow R_{+} \exp [-\mathrm{i} \Delta(\xi-\tau)] .
$$

In addition, for the sake of comparison with standard notation, it is convenient to make the replacements

$$
S_{ \pm} \rightarrow-S_{ \pm} \quad \xi \rightarrow-\xi
$$

so that

$$
\begin{aligned}
& \mathrm{i} \frac{\partial R_{+}}{\partial \tau}+S_{+}+\frac{\Delta}{2} S_{+} S_{-} R_{+}-\frac{1}{2} R_{+} R_{-} S_{+}=0 \\
& -\mathrm{i} \frac{\partial S_{+}}{\partial \xi}+R_{+}+\frac{\Delta}{2} R_{+} R_{-} S_{+}+\frac{1}{2} S_{+} S_{-} R_{+}=0 .
\end{aligned}
$$

The last two terms in each of these two equations have the same order of magnitude with respect to small parameters $\left|R_{ \pm}\right|,\left|S_{ \pm}\right| \ll 1$; but in the limit of strong dynamical Stark shift

$$
|\Delta| \gg 1
$$

the last terms can be neglected and we come to the standard form of the Thirring model equations (12)-(15)

$$
\begin{aligned}
& \mathrm{i} \frac{\partial R_{+}}{\partial \tau}+S_{+}+\frac{\Delta}{2} S_{+} S_{-} R_{+}=0 \\
& -\mathrm{i} \frac{\partial S_{+}}{\partial \xi}+R_{+}+\frac{\Delta}{2} R_{+} R_{-} S_{+}=0 .
\end{aligned}
$$

Let us consider the corresponding limit of the $U-V$ pair of the TPP equations. As was shown in $[4,5]$, the TPP equations can be presented as a compatibility condition of two linear systems,

$$
\frac{\partial \psi}{\partial \tau}=U \psi \quad \frac{\partial \psi}{\partial \xi}=V \psi
$$

where $\psi=\left(\psi_{1}, \psi_{2}\right)^{T}$ and $U, V$ are $2 \times 2$ matrices of the AKNS scheme [20]:

$$
U=\left(\begin{array}{cc}
F & G \\
H & -F
\end{array}\right) \quad V=\left(\begin{array}{cc}
A & B \\
C & -A
\end{array}\right) .
$$

The compatibility condition of two linear systems (18) has the form

$$
\frac{\partial V}{\partial \tau}-\frac{\partial U}{\partial \xi}+V U-U V=0
$$

and leads to the TPP equations (8) if we take

$$
\begin{aligned}
& F=-\mathrm{i} \zeta S_{3} \quad G=(\zeta+\sigma) S_{+} \quad H=(\zeta-\sigma) S_{-} \\
& A=\frac{\mathrm{i}}{2}\left(\Delta+\frac{1}{2 \zeta+\Delta}\right) R_{3} \quad B=-\frac{\zeta+\sigma}{2 \zeta+\Delta} R_{+} \quad C=-\frac{\zeta-\sigma}{2 \zeta+\Delta} R_{-}
\end{aligned}
$$


where the parameter $\sigma$ is connected with $\Delta$ according to

$$
\sigma^{2}=\frac{1}{4}\left(1+\Delta^{2}\right)
$$

and $\zeta$ is an arbitrary spectral parameter.

With the use of the approximations (10) and (11) and accounting for the replacements (13) and (14), we obtain from (21) and (22) the matrix elements of the new $U-V$ pair,

$$
\begin{array}{lrl}
F=\mathrm{i} \lambda^{2}+\frac{\mathrm{i} \Delta}{4} S_{+} S_{-} & G=\lambda^{2} S_{+} \quad H=\Delta S_{-} \\
A=-\frac{\mathrm{i}}{4 \lambda^{2}}-\frac{\mathrm{i} \Delta}{4} R_{+} R_{-} & B=\frac{1}{2} R_{+} \quad C=\frac{\Delta}{2 \lambda^{2}} R_{-}
\end{array}
$$

where the new spectral parameter $\lambda$ is introduced according to definition

$$
\zeta=-\frac{\Delta}{2}-\lambda^{2}
$$

(we shall not use notation $\lambda_{F}$ for wavelength anymore). It is easy to check that the compatibility condition (20) with matrix elements (24), (25) corresponds to the Thirring equations (17). The integrability of Thirring equations was first established in [14], but the $U-V$ pair found in [14] was different from (24), (25). The origin of this ambiguity is discussed in the appendix.

Thus we have found that the classical Thirring model in its original form has an optical application as a corresponding limit of the TPP or SRS equations $\dagger$. Now we shall study the periodic solutions of the Thirring model equations.

\section{Periodic solutions of Thirring model equations}

\subsection{General periodic solutions}

The periodic problem for the Thirring model was first discussed in [22]. Here we derive it in a more effective form with the use of the method suggested in [18].

The simplest approach [23-25] to the finite-gap method is based on the introduction of the 'squared basic functions' built from two basic solutions, $\psi=\left(\psi_{1}, \psi_{2}\right)$ and $\phi=\left(\phi_{1}, \phi_{2}\right)$, of linear systems (18) according to definitions

$$
f=-\frac{1}{2} \mathrm{i}\left(\psi_{1} \phi_{2}+\psi_{2} \phi_{1}\right) \quad g=\psi_{1} \phi_{1} \quad h=-\psi_{2} \phi_{2} .
$$

The functions $\psi, \phi$ satisfy different boundary conditions (in the nonperiodic case they have opposite asymptotics at $\tau \rightarrow \infty$ ), so that $f, g$ and $h$ are algebraic functions of the spectral parameter $\lambda$ and their evolution with change of $\tau$ and $\xi$ is governed by the following systems of linear equations

$$
\begin{aligned}
\partial f / \partial \tau & =-\mathrm{i} H g+\mathrm{i} G h & \partial f / \partial \xi & =-\mathrm{i} C g+\mathrm{i} B h \\
\partial g / \partial \tau & =2 \mathrm{i} G f+2 F g & \partial g / \partial \xi & =2 \mathrm{i} B f+2 A g \\
\partial h / \partial \tau & =-2 \mathrm{i} H f-2 F h & \partial h / \partial \xi & =-2 \mathrm{i} C f-2 A h
\end{aligned}
$$

which are consequences of (18), (19).

The functions (27) can be considered as spherical components of the vector whose length is conserved during the evolution governed by the equations (28). That is, the quantity

$$
f^{2}-g h=P(\lambda)
$$

$\dagger$ It is worth noting that the application of the Thirring model to optical solitons in a fibre was discussed in [21]. 
does not depend on $\xi$ and $\tau$, so that $P$ is function of $\lambda$ only. The periodic solutions are distinguished by the condition that $P=P(\lambda)$ be a polynomial in $\lambda$ [23-25]. Seeking solutions of systems (28) in the form of polynomials in $\lambda$, it is easy to find that the functions $f, g, h$ can contain only even degrees of $\lambda$. Consequently, $P(\lambda)$ is also a polynomial in $\lambda$ containing only even degrees of $\lambda$. The single-phase solution corresponds, as we shall see, to the eight-degree polynomial $P(\lambda)$

$$
P(\lambda)=\prod_{i=1}^{4}\left(\lambda^{2}-\lambda_{i}^{2}\right)=\lambda^{8}-s_{1} \lambda^{6}+s_{2} \lambda^{4}-s_{3} \lambda^{2}+s_{4}
$$

where $\pm \lambda_{i}$ are the zeros of the polynomial. Then the systems (28) give the solution

$f=\lambda^{4}-f_{1} \lambda^{2}+f_{2} \quad g=-\lambda^{2} S_{+}\left(\lambda^{2}-\mu\right) \quad h=-\Delta S_{-}\left(\lambda^{2}-\mu^{*}\right)$

provided $f_{1}, f_{2}, \mu, \mu^{*}$ satisfy the equations

$$
\begin{aligned}
& \frac{\partial S_{+}}{\partial \tau}=2 \mathrm{i}\left(f_{1}-\mu+\frac{\Delta}{4} S_{+} S_{-}\right) S_{+} \quad \frac{\partial S_{+}}{\partial \xi}=-\mathrm{i} R_{+}-\frac{\mathrm{i} \Delta}{2} R_{+} R_{-} S_{+} \\
& \frac{\partial f_{1}}{\partial \tau}=\mathrm{i} \Delta S_{+} S_{-}\left(\mu-\mu^{*}\right) \quad \frac{\partial f_{1}}{\partial \xi}=-\frac{\mathrm{i} \Delta}{2}\left(S_{+} R_{-}-S_{-} R_{+}\right) \\
& f_{2}=\text { constant } \quad \mu=2 f_{2} \frac{R_{+}}{S_{+}} \\
& \frac{\partial\left(S_{+} \mu\right)}{\partial \tau}=2 \mathrm{i} S_{+} f_{2}+\frac{\mathrm{i} \Delta}{2} S_{+}^{2} S_{-} \mu \quad \frac{\partial\left(S_{+} \mu\right)}{\partial \xi}=-\mathrm{i} R_{+} f_{1}+\frac{\mathrm{i}}{2} S_{+}-\frac{\mathrm{i} \Delta}{2} R_{+} R_{-} S_{+} \mu .
\end{aligned}
$$

On substituting (32) and (33) into (35), we find the evolution equations for the parameter $\mu$

$$
\frac{\partial \mu}{\partial \tau}=2 \mathrm{i} f\left(\mu^{1 / 2}\right)=2 \mathrm{i} \sqrt{P\left(\mu^{1 / 2}\right)} \quad \frac{\partial \mu}{\partial \xi}=\frac{1}{4 f_{2}} \frac{\partial \mu}{\partial \tau} .
$$

Thus, $\mu$ depends only on the phase

$$
W=\frac{1}{2}\left(\tau+\frac{\xi}{4 f_{2}}\right) \quad \frac{\mathrm{d} \mu}{\mathrm{d} W}=4 \mathrm{i} \sqrt{P\left(\mu^{1 / 2}\right)}
$$

and moves along some curve in the complex plane as $W$ varies. This curve is determined implicitly by constraint (29) and it is convenient to find it explicitly [18, 19]. Substitution of (30) and (31) into (29) leads to the identity

$$
\left(\lambda^{4}-f_{1} \lambda^{2}+f_{2}\right)^{2}-\Delta S_{+} S_{-} \lambda^{2}\left(\lambda^{2}-\mu\right)\left(\lambda^{2}-\mu^{*}\right)=\lambda^{8}-s_{1} \lambda^{6}+s_{2} \lambda^{4}-s_{3} \lambda^{2}+s_{4} .
$$

We see that the parameter

$$
v=\Delta S_{+} S_{-}=\Delta\left|S_{+}\right|^{2}
$$

can be chosen as a coordinate along the locus of $\mu$. Comparing the coefficients of $\lambda^{k}$ on both sides of (38), we obtain the system

$$
\begin{aligned}
& 2 f_{1}+v=s_{1} \quad f_{1}^{2}+2 f_{2}+v\left(\mu+\mu^{*}\right)=s_{2} \\
& 2 f_{1} f_{2}+v \mu \mu^{*}=s_{3} \quad f_{2}^{2}=s_{4}
\end{aligned}
$$

which coincides with the corresponding system for so-called derivative nonlinear Schrödinger equation [18], so we can use the solution found there

$$
\begin{aligned}
& f_{2}= \pm \sqrt{s_{4}} \\
& \mu(v)=\frac{1}{8 v}\left[4 s_{2} \pm 8 \sqrt{s_{4}}-\left(v-s_{1}\right)^{2}+\mathrm{i} \sqrt{-\mathcal{R}(v)}\right]
\end{aligned}
$$


where the 'resolvent' $\mathcal{R}(v)$ is a fourth-degree polynomial in $v$ :

$$
\begin{aligned}
\mathcal{R}(v)=v^{4}- & 4 s_{1} v^{3}+\left(6 s_{1}-8 s_{2} \pm 48 \sqrt{s_{4}}\right) v^{2} \\
& -\left(4 s_{1}^{3}-16 s_{1} s_{2}+64 s_{3} \pm 32 s_{1} \sqrt{s_{4}}\right) v+\left(-s_{1}^{2}+4 s_{2} \pm 8 \sqrt{s_{4}}\right)^{2} .
\end{aligned}
$$

The zeros $v_{i}, i=1,2,3,4$, of this polynomial are related to the zeros of $P(\lambda)$ by simple symmetric formulae [18]: the zeros

$$
\begin{array}{ll}
v_{1}=\left(\lambda_{1}+\lambda_{2}+\lambda_{3}-\lambda_{4}\right)^{2} & v_{2}=\left(\lambda_{1}+\lambda_{2}-\lambda_{3}+\lambda_{4}\right)^{2} \\
v_{3}=\left(\lambda_{1}-\lambda_{2}+\lambda_{3}+\lambda_{4}\right)^{2} & v_{4}=\left(-\lambda_{1}+\lambda_{2}+\lambda_{3}+\lambda_{4}\right)^{2}
\end{array}
$$

correspond to the upper sign in (43), and the zeros

$$
\begin{array}{ll}
v_{1}=\left(\lambda_{1}+\lambda_{2}+\lambda_{3}+\lambda_{4}\right)^{2} & v_{2}=\left(\lambda_{1}+\lambda_{2}-\lambda_{3}-\lambda_{4}\right)^{2} \\
v_{3}=\left(\lambda_{1}-\lambda_{2}+\lambda_{3}-\lambda_{4}\right)^{2} & v_{4}=\left(-\lambda_{1}+\lambda_{2}+\lambda_{3}-\lambda_{4}\right)^{2}
\end{array}
$$

correspond to the lower sign.

Equations (33) give us

$$
\frac{\mathrm{d} \nu}{\mathrm{d} W}=4 \mathrm{i} v\left(\mu^{*}-\mu\right)
$$

and with the use of (42) we obtain

$$
\frac{\mathrm{d} v}{\mathrm{~d} W}=\sqrt{-\mathcal{R}(v)}
$$

This equation can easily be resolved by means of elliptic functions. If $v$ is known, the field $S_{+}(\xi, \tau)$ can be found from the equations

$$
\frac{\mathrm{d} S_{+}}{\mathrm{d} \tau}=\frac{\mathrm{i}}{2}\left(2 s_{1}-v-4 \mu\right) S_{+} \quad \frac{\mathrm{d} S_{+}}{\mathrm{d} \xi}=\frac{\mathrm{i}}{2}\left(s_{1} \pm \frac{s_{3}}{\sqrt{s_{4}}}\right) S_{+}+4 f_{2} \frac{\partial S_{+}}{\partial \xi}
$$

which yield

$$
S_{+}(\xi, \tau)=\exp \left[\frac{\mathrm{i}}{2}\left(s_{1} \pm \frac{s_{3}}{\sqrt{s_{4}}}\right) \tau\right] \widetilde{S}_{+}(W)
$$

where $\widetilde{S}_{+}(W)$ must satisfy the equation

$$
\frac{\mathrm{d} \widetilde{S}_{+}}{\mathrm{d} W}=\frac{\mathrm{i}}{2}\left(2 s_{1}-v-4 \mu\right) \widetilde{S}_{+} .
$$

In the following we can suppose without loss of generality that $\Delta \geqslant 0$ and, hence, $v \geqslant 0$, too. It is clear that the zeros $\lambda_{i}$ must be numbers such that $v$ oscillates between two positive zeros of the resolvent $\mathcal{R}(v)$. The polynomial $\mathcal{R}(v)$ has four zeros $v_{i}$ given by (44) or (45) depending on a choice of a sign in (43). We shall consider only the cases when all $\nu_{i}$ are real. It is convenient to enumerate $\lambda_{i}$ so that

$$
v_{1} \geqslant v_{2} \geqslant v_{3} \geqslant v_{4} \text {. }
$$

Let us note the important particular cases.

(i) The zeros $\lambda_{i}$ consist of two complex conjugate pairs

$$
\lambda_{1}=\alpha+\mathrm{i} \gamma \quad \lambda_{2}=\alpha-\mathrm{i} \gamma \quad \lambda_{3}=\beta-\mathrm{i} \delta \quad \lambda_{4}=\beta+\mathrm{i} \delta .
$$

Then (45) yields

$v_{1}=4(\alpha+\beta)^{2} \quad v_{2}=4(\alpha-\beta)^{2} \quad v_{3}=-4(\gamma-\delta)^{2} \quad v_{4}=-4(\gamma+\delta)^{2}$

where the variable $v$ oscillates in the interval $v_{1} \geqslant v \geqslant v_{2}$, and (44) results in the complex values of $v_{i}$. 
(ii) All four $\lambda_{i}$ are real and

$$
\lambda_{1} \geqslant \lambda_{2} \geqslant \lambda_{3} \geqslant \lambda_{4} \text {. }
$$

Both (44) and (45) yield the real and positive $v_{i}$ corresponding to different periodic solutions for which the variable $v$ oscillates in the intervals $v_{1} \geqslant v \geqslant v_{2}$ or $v_{3} \geqslant v \geqslant v_{4}$.

We now turn to finding the periodic solutions. Let us first discuss the case when the variable $v$ oscillates in the interval $v_{1} \geqslant v \geqslant v_{2}$. We choose initial conditions so that $v=v_{1}$ at $W=0$. Then equation (46) leads to the solution

$$
\begin{aligned}
v & =\Delta\left|S_{+}\right|^{2} \\
& =\frac{\left(v_{2}-v_{4}\right) v_{1}+\left(v_{1}-v_{2}\right) v_{4} \operatorname{sn}^{2}\left(\sqrt{\left(v_{1}-v_{3}\right)\left(v_{2}-v_{4}\right)} W / 2, m\right)}{v_{2}-v_{4}+\left(v_{1}-v_{2}\right) \operatorname{sn}^{2}\left(\sqrt{\left(v_{1}-v_{3}\right)\left(v_{2}-v_{4}\right)} W / 2, m\right)}
\end{aligned}
$$

where the elliptic function parameter is given by

$$
m=\frac{\left(v_{1}-v_{2}\right)\left(v_{3}-v_{4}\right)}{\left(v_{1}-v_{3}\right)\left(v_{2}-v_{4}\right)} \text {. }
$$

This gives the squared modulus of the field component $S_{+}$, and equations (32) and (40) yield an expression for squared modulus of $R_{+}$component

$$
\left|R_{+}\right|^{2}=\frac{1}{4 s_{4} \Delta}\left[s_{3} \mp \sqrt{s_{4}}\left(s_{1}-v\right)\right] .
$$

The following calculations take a more convenient form in terms of the Weierstrass elliptic functions. Let us introduce the zeros of the Weierstrass cubic by means of the following expressions

$$
\begin{aligned}
& e_{1}=\frac{1}{12}\left[2\left(v_{1}-v_{3}\right)\left(v_{2}-v_{4}\right)-\left(v_{1}-v_{2}\right)\left(v_{3}-v_{4}\right)\right] \\
& e_{2}=\frac{1}{12}\left[2\left(v_{1}-v_{2}\right)\left(v_{3}-v_{4}\right)-\left(v_{1}-v_{3}\right)\left(v_{2}-v_{4}\right)\right] \\
& e_{3}=-\frac{1}{12}\left[\left(v_{1}-v_{3}\right)\left(v_{2}-v_{4}\right)+\left(v_{1}-v_{2}\right)\left(v_{3}-v_{4}\right)\right] .
\end{aligned}
$$

Then equation (53) can be written in the form

$$
v=v_{1} \frac{\wp(W)-\wp(\rho)}{\wp(W)-\wp(\kappa)}
$$

where the parameters $\kappa$ and $\rho$ are defined by the expressions

$\wp(\kappa)=e_{3}-\frac{1}{4}\left(v_{1}-v_{2}\right)\left(v_{1}-v_{3}\right) \quad \wp(\rho)=e_{3}-\frac{v_{4}}{4 v_{1}}\left(v_{1}-v_{2}\right)\left(v_{1}-v_{3}\right)$.

After substitution of (57), (42) and (46) into (48), one can integrate the equation with the use of the formula

$$
\int \frac{\mathrm{d} z}{\wp(z)-\wp(\kappa)}=\frac{1}{\wp^{\prime}(\kappa)}\left[2 z \zeta(\kappa)+\ln \frac{\sigma(z-\kappa)}{\sigma(z+\kappa)}\right]
$$

where $\zeta$ and $\sigma$ are the Weierstrass functions. After a simple calculation, we get the expression for the $S_{+}$component

$$
\begin{aligned}
S_{+}(\xi, \tau)=\sqrt{\frac{\nu_{1}}{\Delta}} \exp \left[\mathrm{i}\left(\frac{3}{2} s_{1}-\frac{\nu_{1}}{2}-\frac{\sqrt{\nu_{1} \nu_{2} \nu_{3} \nu_{4}}}{2 \nu_{1}}\right) W+(\zeta(\rho)-\zeta(\kappa)) W\right. \\
\left.+\frac{\mathrm{i}}{2}\left(s_{1} \pm \frac{s_{3}}{\sqrt{s_{4}}}\right) \tau\right] \frac{\sigma(\kappa) \sigma(W-\rho)}{\sigma(\rho) \sigma(W-\kappa)} \quad W=\frac{1}{2}\left(\tau \pm \frac{\xi}{4 \sqrt{s_{4}}}\right) .
\end{aligned}
$$


The case when $v$ oscillates in the interval $v_{3} \geqslant v \geqslant v_{4}$ can be considered in the same way. Initial conditions are chosen so that $v=v_{4}$ at $W=0$. For $v$ we get the expression

$v=\Delta\left|S_{+}\right|^{2}=\frac{\left(v_{1}-v_{3}\right) v_{4}+\left(v_{3}-v_{4}\right) v_{1} \operatorname{sn}^{2}\left(\sqrt{\left(\nu_{1}-v_{3}\right)\left(v_{2}-v_{4}\right)} W / 2, m\right)}{v_{1}-v_{3}+\left(v_{3}-v_{4}\right) \operatorname{sn}^{2}\left(\sqrt{\left(v_{1}-v_{3}\right)\left(v_{2}-v_{4}\right)} W / 2, m\right)}$

or in terms of Weierstrass functions,

$$
v=v_{4} \frac{\wp(W)-\wp(\omega)-\wp(\kappa)}{\wp(W)}
$$

where $\kappa$ and $\rho$ are now defined by

$\wp(\kappa)=e_{3}-\frac{1}{4}\left(v_{2}-v_{4}\right)\left(v_{3}-v_{4}\right) \quad \wp(\rho)=e_{3}-\frac{v_{1}}{4 v_{4}}\left(v_{2}-v_{4}\right)\left(v_{3}-v_{4}\right)$.

The corresponding periodic solution of the Thirring model equations takes the form

$$
\begin{aligned}
S_{+}(\xi, \tau)=\sqrt{\frac{\nu_{4}}{\Delta}} \exp \left[\mathrm{i}\left(\frac{3}{2} s_{1}-\frac{\nu_{1}}{2}-\frac{\sqrt{\nu_{1} \nu_{2} \nu_{3} v_{4}}}{2 v_{4}}\right) W-(\zeta(\rho)-\zeta(\kappa)) W\right. \\
\left.+\frac{\mathrm{i}}{2}\left(s_{1} \pm \frac{s_{3}}{\sqrt{s_{4}}}\right) \xi\right] \frac{\sigma(\kappa) \sigma(W+\rho)}{\sigma(\rho) \sigma(W+\kappa)} \quad W=\frac{1}{2}\left(\tau \pm \frac{\xi}{4 \sqrt{s_{4}}}\right) .
\end{aligned}
$$

The formulae obtained give the general periodic solutions of the Thirring model equations.

\subsection{Soliton limit}

Let us consider the soliton limit of the solution (60), when $v_{2}=v_{3}$, i.e.

$e_{1}=e_{2}=a=\frac{1}{12}\left(v_{1}-v_{2}\right)\left(v_{2}-v_{4}\right) \quad e_{3}=-2 a=-\frac{1}{6}\left(v_{1}-v_{2}\right)\left(v_{2}-v_{4}\right)$.

By means of the well known limiting expressions for the Weierstrass function, we obtain from (60)

$$
\begin{gathered}
S_{+}(\xi, \tau)=\frac{1}{2 \sqrt{\Delta}} \exp \left[\mathrm{i}\left(\frac{3}{2} s_{1}-\frac{\nu_{2}}{2}-\frac{\sqrt{\nu_{1} v_{4}}}{2}\right) W+\frac{\mathrm{i}}{2}\left(s_{1} \pm \frac{s_{3}}{\sqrt{s_{4}}}\right) \tau\right] \\
\times \sqrt{v_{1}} \frac{\sinh (\sqrt{3 a} W) \operatorname{coth}(\sqrt{3 a} \rho)+\cosh (\sqrt{3 a} W)}{\sinh (\sqrt{3 a} W) \operatorname{coth}(\sqrt{3 a} \kappa)+\cosh (\sqrt{3 a} W)} .
\end{gathered}
$$

Equations (63) give in this limit

$\sqrt{3 a} \operatorname{coth}(\sqrt{3 a} \kappa)=-\frac{\mathrm{i}}{2}\left(v_{1}-v_{2}\right) \quad \sqrt{3 a} \operatorname{coth}(\sqrt{3 a} \rho)=-\frac{\mathrm{i}}{2} \sqrt{\frac{v_{4}}{v_{1}}}\left(v_{1}-v_{2}\right)$.

Let us denote

$$
2 \theta=\sqrt{3 a} W \quad \cos ^{2} \frac{\eta}{2}=\frac{v_{2}-v_{4}}{v_{1}-v_{4}}
$$

so that

$$
\operatorname{coth}(\sqrt{3 a} \kappa)=-\mathrm{i} \tan \frac{\eta}{2} \quad \operatorname{coth}(\sqrt{3 a} \rho)=-\mathrm{i} \sqrt{\frac{\nu_{4}}{v_{1}}} \tan \frac{\eta}{2}
$$

and (66) can be transformed to the form

$$
\begin{aligned}
S_{+}(\xi, \tau)=\frac{1}{2 \sqrt{\Delta}} \exp \left[\mathrm{i}\left(\frac{3}{2} s_{1}-\frac{\nu_{2}}{2}-\frac{\sqrt{\nu_{1} \nu_{4}}}{2}\right) W+\frac{\mathrm{i}}{2}\left(s_{1} \pm \frac{s_{3}}{\sqrt{s_{4}}}\right) \tau\right] \\
\times\left[\sqrt{\nu_{1}}+\sqrt{\nu_{4}}+\left(\sqrt{\nu_{1}}-\sqrt{\nu_{4}}\right) \frac{\cosh (2 \theta+\mathrm{i} \eta / 2)}{\cosh (2 \eta-\mathrm{i} \eta / 2)}\right] .
\end{aligned}
$$


This solution describes soliton propagating on a constant background which can vanish for some special cases.

The analogous soliton limit of the solution (64) has the form

$$
\begin{aligned}
S_{+}(\xi, \tau)=\frac{1}{2 \sqrt{\Delta}} \exp \left[\mathrm{i}\left(\frac{3}{2} s_{1}-\frac{\nu_{2}}{2}-\frac{\sqrt{\nu_{1} v_{4}}}{2}\right) W+\frac{\mathrm{i}}{2}\left(s_{1} \pm \frac{s_{3}}{\sqrt{s_{4}}}\right) \tau\right] \\
\times\left[\sqrt{\nu_{1}}+\sqrt{\nu_{4}}-\left(\sqrt{\nu_{1}}-\sqrt{\nu_{4}}\right) \frac{\cosh (2 \theta-\mathrm{i} \eta / 2)}{\cosh (2 \eta+\mathrm{i} \eta / 2)}\right] .
\end{aligned}
$$

Consider some particular cases. Let

$$
\lambda_{1}=\lambda_{4}=\alpha+\mathrm{i} \gamma \quad \lambda_{2}=\lambda_{3}=\alpha-\mathrm{i} \gamma
$$

so that equations (45) (corresponding to the lower sign in above formulae) give

$$
v_{1}=16 \alpha^{2} \quad v_{2}=v_{3}=0 \quad v_{4}=-16 \gamma^{2}
$$

and

$$
\cos ^{2} \frac{\eta}{2}=\frac{\gamma^{2}}{\alpha^{2}+\gamma^{2}}
$$

It is convenient to introduce the parametrization

$$
\alpha=D \sin \frac{\eta}{2} \quad \gamma=D \cos \frac{\eta}{2} .
$$

Substitution of these expressions into (69) leads to the soliton solution

$$
S_{+}=\frac{2 D}{\sqrt{\Delta}} \exp [\mathrm{i} \Phi(\xi, \tau)] \frac{\sin \eta}{\cosh (2 \theta-\mathrm{i} \eta / 2)}
$$

where $\theta=\sin \eta\left(D^{2} \tau+\xi / D^{2}\right)$ and $\Phi(\xi, \tau)$ is some phase which we are not interested in. The 'intensity' equals

$$
\left|S_{+}\right|^{2}=\frac{8 D^{2} \sin ^{2} \eta}{\Delta} \frac{1}{\cosh 4 \theta+\cos \eta} .
$$

This soliton solution can be obtained as an appropriate limit of the TPP soliton found in [5]. Indeed, the TPP soliton solution is parametrized by the eigenvalue

$$
\zeta=\alpha_{1}+\mathrm{i} \gamma_{1}
$$

of the corresponding scattering problem (18), (19) and has the form

$$
S_{3}-1=\frac{2 \gamma_{1} \sin 2 \chi}{\sigma} \frac{1}{\cosh \left(4 \gamma_{1} W\right)+\cos 2 \chi}
$$

where the parameter $\chi$ is defined by the relation

$$
\tan \chi=\frac{2 \sigma \gamma_{1}}{\sigma^{2}-\alpha_{1}^{2}-\gamma_{1}^{2}} .
$$

The phase $W$ is equal to

$$
W=\tau-\xi /\left(4\left[\left(\alpha_{1}+\Delta / 2\right)^{2}+\gamma_{1}\right]\right)
$$

and $\sigma$ is given by (22). Taking into account (11), (14), (26) and (74), we rewrite the formula (77) by means of identifications

$$
\begin{aligned}
& \alpha_{1}+\Delta / 2=-\operatorname{Re}\left(\lambda^{2}\right)=-\left(\alpha^{2}-\gamma^{2}\right)=D^{2} \cos \eta \\
& \gamma_{1}=-\operatorname{Im}\left(\lambda^{2}\right)=-2 \alpha \gamma=-D^{2} \sin \eta
\end{aligned}
$$


in the form

$\left|S_{+}\right|^{2}=-\frac{8 D^{2} \sin \eta \sin (2 \chi)}{\sqrt{1+\Delta^{2}}} \frac{1}{\cosh \left[-4 D^{2} \sin \eta\left(\tau+\xi /\left(4 D^{4}\right)\right)\right]+\cos 2 \chi}$.

Since equation (78) rewritten in new parameters

$$
\tan 2 \chi=-\frac{\sqrt{1+\Delta^{2}} D^{2} \sin \eta}{\Delta D^{2} \cos \eta+\frac{1}{4}-D^{4}}
$$

gives in the limit $\Delta \rightarrow \infty$ the relation (at $D^{2} \simeq \frac{1}{4}$ it is enough to have $\Delta^{2} \gg 1$ )

$$
\tan 2 \chi \simeq-\tan \eta \quad \text { that is } 2 \chi \simeq-\eta
$$

it is easy to see that equation (82) immediately reduces to (76) in this limit.

Now let all the $\lambda_{i}$ be real and equal to

$$
\lambda_{1}=\frac{1}{2}(\alpha+\beta) \quad \lambda_{2}=\lambda_{3}=\frac{1}{2} \beta \quad \lambda_{4}=-\frac{1}{2}(\alpha-\beta)
$$

so that

$$
v_{1}=4 \beta^{2} \quad v_{2}=v_{3}=\alpha^{2} \quad v_{4}=0 .
$$

Then the field intensity corresponding to (69) is equal to

$$
\left|S_{+}\right|^{2}=\frac{2 \beta^{2}}{\Delta} \frac{(\cosh 4 \theta+1)(\cos \eta+1)}{\cosh 4 \theta+\cos \eta}
$$

where

$$
\theta=\frac{\sqrt{3 a}}{2} W=\frac{\beta^{2} \sin \eta}{4}\left(\tau+\frac{2 \xi}{\beta^{2}\left(\alpha^{2}-\beta^{2}\right)}\right) .
$$

This is the 'bright' soliton solution on the constant background $\left|S_{+}\right|_{0}^{2}=\alpha^{2} / \Delta$.

Similarly, the intensity of solution (70) in the case (83) takes the form

$$
\left|S_{+}\right|^{2}=\frac{2 \beta^{2}}{\Delta} \frac{(\cosh 4 \theta-1)(1-\cos \eta)}{\cosh 4 \theta+\cos \eta}
$$

which corresponds to the 'dark' soliton solution on the constant background.

\subsection{Whitham equations}

The periodic solutions are parametrized by four variables $\lambda_{i}, i=1,2,3,4$, depending on space and time coordinates in slightly nonuniform and nonstationary problems. The evolution of $\lambda_{i}$ in such problems is governed by the Whitham equations [26]. These equations can be easily derived by well known averaging methods (see, e.g. [19]) and have standard form. Therefore we shall write the final result here. The Whitham equations for $\lambda_{i}$ have the diagonal form

$$
\frac{\partial \lambda_{i}^{2}}{\partial \xi}+\frac{1}{v_{i}} \frac{\partial \lambda_{i}^{2}}{\partial \tau}=0 \quad i=1,2,3,4
$$

where the group velocities are equal to

$$
\frac{1}{v_{i}}=\left(1-\frac{T}{\partial_{i} T} \partial_{i}\right) \frac{1}{V} \quad \partial_{i} \equiv \frac{\partial}{\partial \lambda_{i}} \quad i=1,2,3,4
$$

with period $T$ being given by

$$
T=\frac{1}{2} \oint \frac{\mathrm{d} \mu}{\sqrt{-P(\mu)}}=\frac{2 \mathrm{~K}(m)}{\sqrt{\left(\lambda_{1}^{2}-\lambda_{4}^{2}\right)\left(\lambda_{3}^{2}-\lambda_{2}^{2}\right)}}
$$


where $\mathrm{K}(m)$ is the complete elliptic integral of the first kind and $V$ is the phase velocity of nonlinear periodic wave

$$
V=2 f_{2}= \pm 2 \sqrt{\prod_{i=1}^{4} \lambda_{i}^{2}} .
$$

Note that these equations formally coincide with those for the AB system [27], but in the case under consideration the variables $\lambda_{i}$ can be real, whereas in the AB system case they are complex.

\section{Conclusion}

We have found that TPP and SRS equations can be reduced to the classical Thirring model in one practically important limit and have obtained periodic solutions of the Thirring model equations in an effective form including the corresponding modulation Whitham equations. The analysis of the solutions of these modulation equations is rather complicated and will be published elsewhere.

\section{Acknowledgments}

$\mathrm{AK}$ and $\mathrm{AZ}$ are grateful to Professor $\mathrm{H}$ Paul and the staff of the 'Arbeitsgruppe Nichklassische Strahlung' of the Max Planck Society at the Humboldt University, Berlin (where this work was started) for their kind hospitality. Financial support from DFG grant 436 RUS 113/89/10 (R,S) and RFFI grants 96-02-20026, 96-01-01453, 97-02-17418 is also appreciated.

\section{Appendix}

As we have found in section 2, the Thirring model equations

$$
\frac{\partial S_{+}}{\partial \xi}=-\mathrm{i} R_{+}-\frac{\mathrm{i} \Delta}{2} R_{+} R_{-} S_{+} \quad \frac{\partial R_{+}}{\partial \tau}=\mathrm{i} S_{+}+\frac{\mathrm{i} \Delta}{2} S_{+} S_{-} R_{+}
$$

can be presented as a compatibility condition

$$
\frac{\partial V}{\partial \tau}-\frac{\partial U}{\partial \xi}+[V, U]=0
$$

of two linear systems

$$
\frac{\partial \psi}{\partial \tau}=U \psi \quad \frac{\partial \psi}{\partial \xi}=V \psi
$$

where

$$
\begin{aligned}
U & =\left(\begin{array}{cc}
\mathrm{i} \lambda^{2}+\mathrm{i} \Delta S_{+} S_{-} / 4 & \lambda^{2} S_{+} \\
\Delta S_{-} & -\mathrm{i} \lambda^{2}-\mathrm{i} \Delta S_{+} S_{-} / 4
\end{array}\right) \\
V & =\left(\begin{array}{cc}
-\mathrm{i} /\left(4 \lambda^{2}\right)-\mathrm{i} \Delta R_{+} R_{-} / 4 & R_{+} / 2 \\
\Delta R_{-} /\left(2 \lambda^{2}\right) & \mathrm{i} /\left(4 \lambda^{2}\right)+\mathrm{i} \Delta R_{+} R_{-} / 4
\end{array}\right) .
\end{aligned}
$$


On the other hand, in [14] the $U-V$ pair corresponding to the Thirring model was found in the form

$$
\begin{gathered}
U=\left(\begin{array}{cc}
\mathrm{i} \Delta \lambda^{2} / 4+\mathrm{i} \Delta S_{+} S_{-} / 4 & \Delta \lambda S_{+} / 2 \\
\Delta \lambda S_{-} / 2 & -\mathrm{i} \Delta \lambda^{2} / 4-\mathrm{i} \Delta S_{+} S_{-} / 4
\end{array}\right) \\
V=\left(\begin{array}{cc}
-\mathrm{i} /\left(\Delta \lambda^{2}\right)-\mathrm{i} \Delta R_{+} R_{-} / 4 & R_{+} / \lambda \\
R_{-} / \lambda & \mathrm{i} /\left(\Delta \lambda^{2}\right)+\mathrm{i} \Delta R_{+} R_{-} / 4
\end{array}\right) .
\end{gathered}
$$

Let us consider this ambiguity by means of the prolongation method (see, e.g. [28]).

We shall look for $U$ and $V$ in the form

$V=\mathcal{A}+\mathcal{B} R_{+}+\mathcal{C} R_{-}+\mathcal{D} R_{+} R_{-} \quad U=\mathcal{E}+\mathcal{F} S_{+}+\mathcal{G} S_{-}+\mathcal{H} S_{+} S_{-}$

where $\mathcal{A}, \mathcal{B}, \ldots, \mathcal{H}$ are unknown $2 \times 2$ matrices. On substituting (A6) into (A2) we obtain, with the use of equations (A1), the system

$$
\begin{array}{lcc}
{[\mathcal{A}, \mathcal{E}]=[\mathcal{D}, \mathcal{E}]=[\mathcal{A}, \mathcal{H}]=[\mathcal{D}, \mathcal{H}]=[\mathcal{B}, \mathcal{F}]=[\mathcal{C}, \mathcal{G}]=0} \\
\mathrm{i} \mathcal{F}+[\mathcal{B}, \mathcal{E}]=0 & -\mathrm{i} \mathcal{G}+[\mathcal{C}, \mathcal{E}]=0 & \mathrm{i} \mathcal{B}+[\mathcal{A}, \mathcal{F}]=0 \\
-\mathrm{i} \mathcal{C}+[\mathcal{A}, \mathcal{G}]=0 & -\mathrm{i} \mathcal{D}+\mathrm{i} \mathcal{H}+[\mathcal{B}, \mathcal{G}]=0 & \mathrm{i} \mathcal{D}-\mathrm{i} \mathcal{H}+[\mathcal{C}, \mathcal{F}]=0 \\
-\frac{\mathrm{i} \Delta}{2} \mathcal{C}+[\mathcal{C}, \mathcal{H}]=0 & \frac{\mathrm{i} \Delta}{2} \mathcal{B}+[\mathcal{B}, \mathcal{H}]=0 & -\frac{\mathrm{i} \Delta}{2} \mathcal{G}+[\mathcal{D}, \mathcal{G}]=0 .
\end{array}
$$

This system can be satisfied if we take

$$
\mathcal{E}=e \mathcal{A} \quad \mathcal{D}=d \mathcal{A} \quad \mathcal{H}=h \mathcal{A} \quad \mathcal{F}=f \mathcal{B} \quad \mathcal{G}=g \mathcal{C}
$$

provided the following relations for commutators are fulfilled

$$
\begin{aligned}
& {[\mathcal{A}, \mathcal{B}]=\frac{\mathrm{i} f}{e} \mathcal{B}=-\frac{\mathrm{i}}{f} \mathcal{B}=\frac{\mathrm{i} \Delta}{2 h} \mathcal{B}=-\frac{\mathrm{i} \Delta}{2 d} \mathcal{B}} \\
& {[\mathcal{A}, \mathcal{C}]=-\frac{\mathrm{i} g}{e} \mathcal{C}=\frac{\mathrm{i}}{g} \mathcal{C}=-\frac{\mathrm{i} \Delta}{2 h} \mathcal{C}=\frac{\mathrm{i} \Delta}{2 d} \mathcal{C}} \\
& {[\mathcal{B}, \mathcal{C}]=\frac{\mathrm{i}(d-h)}{g} \mathcal{A}=\frac{\mathrm{i}(d-h)}{f} \mathcal{A} .}
\end{aligned}
$$

That is

$$
f^{2}=g^{2}=-e \quad g=f \quad d=-h=\frac{\Delta f}{2}
$$

and (A8) can be written in the form

$$
[\mathcal{A}, \mathcal{B}]=-\frac{\mathrm{i}}{f} \mathcal{B} \quad[\mathcal{A C}]=\frac{\mathrm{i}}{f} \mathcal{C} \quad[\mathcal{B}, \mathcal{C}]=\mathrm{i} \Delta \mathcal{A}
$$

It easy to see that these relations can be satisfied by means of the matrices $\sigma_{ \pm}=\left(\sigma_{1} \pm \mathrm{i} \sigma_{2}\right) / 2$ and $\sigma_{3}$, where $\sigma_{1}, \sigma_{2}, \sigma_{3}$ are the Pauli matrices. Indeed, if we choose

$$
\mathcal{A}=\alpha \sigma_{3} \quad \mathcal{B}=\beta \sigma_{+} \quad \mathcal{C}=\gamma \sigma_{-}
$$

then we must have

$$
f=\frac{\Delta}{\beta \gamma} \quad \alpha=-\frac{\mathrm{i}}{\Delta} \beta \gamma
$$

which leads to the $U-V$ pair with two spectral parameters $\beta$ and $\gamma$ :

$$
\begin{gathered}
U=\left(\begin{array}{cc}
\mathrm{i} \Delta /(4 \beta \gamma)+\mathrm{i} \Delta S_{+} S_{-} / 4 & \Delta S_{+} /(2 \gamma) \\
\Delta S_{-} /(2 \beta) & -\mathrm{i} \Delta /(4 \beta \gamma)-\mathrm{i} \Delta S_{+} S_{-} / 4
\end{array}\right) \\
V=\left(\begin{array}{cc}
-\mathrm{i} \beta \gamma / \Delta-\mathrm{i} \Delta R_{+} R_{-} / 4 & \beta R_{+} \\
\gamma R_{-} & \mathrm{i} \beta \gamma / \Delta+\mathrm{i} \Delta R_{+} R_{-} / 4
\end{array}\right) .
\end{gathered}
$$


At $\beta=\frac{1}{2}, \gamma=\Delta /\left(2 \lambda^{2}\right)$ we obtain the $U-V$ pair in the form (A4), and at $\beta=\gamma=1 / \lambda$ we return to (A5). Thus, the ambiguity under consideration arises due to the possibility of two spectral parameters in the $U-V$ representation of the classical Thirring model.

\section{References}

[1] Maimistov A I, Basharov A M, Elyutin S O and Sklyarov Yu M 1990 Phys. Rep. 1911

[2] Kittelman O, Ringling J, Nazarkin A, Korn G and Hertel I V 1996 Phys. Rev. Lett. 762682

[3] Chilukuri S 1996 Phys. Rev. A 54908

[4] Kaup D J 1983 Physica 6D 143

[5] Steudel H 1983 Physica 6D 155

[6] Meinel R 1984 Opt. Commun. 49224

[7] Zabolotskii A A 1994 Phys. Rev. A 503384

[8] Zabolotskii A A 1995 Zh. Eksp. Teor. Fiz. 1071100

[9] Kamchatnov A M 1996 Zh. Eksp. Teor. Fiz. 109786

[10] Kamchatnov A M and Ginovart F 1996 J. Phys. A: Math. Gen. 294127

[11] Tan-no N, Yokoto K and Inaba H 1972 Phys. Rev. Lett. 291211

[12] Thirring W 1958 Ann. Phys., NY 391

[13] Steudel H 1977 Ann. Phys., Lpz. 34188

[14] Mikhailov A V 1976 Pis. Zh. Eksp. Teor. Fiz. 23356

[15] Kuznetsov E A and Mikhailov A V 1977 Teor. Mat. Fiz. 30303

[16] Zakharov V E, Manakov S V, Novikov S P and Pitaevskii L P 1980 The Theory of Solitons (Moscow: Nauka)

[17] Belokolos E D, Bobenko A I, Enol'skii V Z, Its A R and Matveev V B 1994 Algebro-Geometric Approach to Nonlinear Integrable Equations (Berlin: Springer)

[18] Kamchatnov A M 1990 J. Phys. A: Math. Gen. 232945

[19] Kamchatnov A M 1997 Phys. Rep. 286199

[20] Ablowitz M J, Kaup D J, Newell A C and Segur H 1974 Stud. Appl. Math. 53241

[21] Aceves A B and Wabnitz S 1989 Phys. Lett. A 14137

[22] Bikbaev R F 1985 Teor. Mat. Fiz. 63377

[23] Kozel V A and Kotlyarov V P 1976 DAN UkrSSR 11879

[24] Its A R and Kotlyarov V P 1976 DAN UkrSSR 11965

[25] Forest M G and McLaughlin D W 1982 J. Math. Phys. 271248

[26] Whitham G B 1974 Linear and Nonlinear Waves (New York: Wiley)

[27] Kamchatnov A M and Pavlov M V 1995 J. Phys. A: Math. Gen. 283279

[28] Kaup D J 1980 Physica 1D 391 